\title{
Towards built-in capture-recapture mixed models in program E-SURGE
}

\author{
R. Choquet* and O. Gimenez
}

Received: date / Accepted: date

\begin{abstract}
We consider the first steps towards implementing capture-recapture mixed models in program E-SURGE. The main issue when estimating the parameters of mixed models is that integrals associated with the random effects distributions need to be dealt with. Rather than using a Bayesian approach with Markov chain Monte Carlo and in line with Gimenez and Choquet (2010), we show that a frequentist approach using numerical integration can be tractable when independent clusters of individuals can be identified. In this case, the maximum likelihood approach is time-efficient because the dimension of the integral for the likelihood is small. This allows us to integrate the likelihood by an efficient and appropriate quadrature method with a procedure for error control. Building on program E-SURGE (Choquet, Rouan, and Pradel, 2009), we extend the GEMACO language (Choquet, 2008) to incorporate random effects in a large set of capture-recapture models, including multievent models (Pradel, 2005). To illustrate the flexible implementation of mixed models in ESURGE, we consider two real examples, one with an individual random effect and one with group random effects. Future developments and limitations are also discussed.
\end{abstract}

\section{Keywords}

Adaptive method, Cluster of individuals, Gauss-Hermite quadrature, GLMM, Hidden Markov chain, Normal random effects, State uncertainty.

\section{Introduction}

With the improvement of Markov Chain Monte-Carlo (MCMC algorithms), the development of new software (e.g. Winbugs, Lunn et al. (2000)) and the availability of more and more powerful computers, mixed models are becoming more and more popular in ecological

Rémi Choquet

CEFE, campus CNRS, UMR 5175, 1919 Route de Mende, Montpellier cedex 5,

F-34293, France.

E-mail: remi.choquet@cefe.cnrs.fr

Olivier Gimenez

CEFE, campus CNRS, UMR 5175, 1919 Route de Mende, Montpellier cedex 5,

F-34293, France. 
science (Clark, 2005), and particularly in capture-recapture (CR) (Wintrebert et al., 2005; Link and Barker, 2005; Gimenez et al., 2009). A non exhaustive list of advantages is:

- The possibility to explicitly decompose the variance into several components.

- The possibility to model dependence among parameters or random effects.

- That boundary estimates are avoided for small sample size data sets.

In the CR research area, mixed models remain under used despite their potential. One reason is that practitioners lack sufficient statistical training to code (or pseudocode in the case of WinBUGS) Bayesian implementations of mixed effects models. In non-Bayesian applications, Burnham and Anderson (2002) and Royle and Link (2002) considered the use of shrinkage estimator for time random effects. However, the mandatory assumption of non-boundary time fixed effect estimates are often violated, making this approach difficult to implement for a general class of model. Moreover, it is not applicable to the scale of individual effect as individual fixed effects estimates are not available. Another reason is with computation challenges in non-Bayesian applications such as numerical integration. This paper addresses the latter situation.

In this paper, we propose a first step toward implementing mixed CR models in E-SURGE. We will only consider a simple case with independent and identically distributed random effects. We use the independence property to reduce the dimension of the integral associated with the marginal likelihood. Thus because fitting problems become more and more complex, in the ideal case, we would like to consider an adaptive approach where the selected algorithm is suited to the model of interest. Developing adapted algorithms has proven in the past to be efficient in M-SURGE (Choquet et al., 2004) to deal with models with complex age structures. The situation here is even more challenging and the road will be quite long before achieving this goal.

In line with Coull and Agresti (1999), McClintock et al. (2009), Gimenez and Choquet (2010), we consider a numerical approximation using Gauss-Hermite quadrature to calculate the marginal likelihood which has no analytical solution in presence of random effects. Gaussian quadrature is known to work efficiently for low dimension integrals over a large class of problems (Lemenuel-Diot et al., 2005; Heiss and Winschel, 2008; Arndt et al., 2006). Numerical integration using Gauss-Hermite quadrature was used in Gimenez and Choquet (2010) to fit the Cormack-Jolly-Seber (CJS) model with individual random effects. We implement this approach for the set of multievent model (Pradel, 2005), extensions of multistate CR models to handle state uncertainty, which are already implemented in ESURGE (Choquet, Rouan, and Pradel, 2009). In addition, we have considered an adaptive Gauss-Hermite quadrature to get efficient algorithms for group random effects. To get built-in random effects, we extend also the language in GEMACO (Choquet, 2008) to automatically build matrices of constraints: Mixed models can be specified with simple phrases.

We show how E-SURGE makes it is easy to fit mixed models using two applications. The first application illustrates a model accounting for a potential effect of the observation process with individual random effects applied to an European Dipper data set. The second application illustrates a model with group random effects applied to a Bank Vole data set. 
2 Notations

$K$ is the number of occasions,

$N I$ is the number of individuals,

$G$ is the number of groups,

$\mathbf{h}$ is the data set of capture histories,

$I_{S}$ is the identity matrix of size $s$,

$\mathscr{N}(\mu, \sigma)$ is the normal distribution with mean $\mu$ and variance $\sigma$,

$\Theta$ is the vector of biological parameters, $\Theta_{i}$ the vector of biological parameters for individual $i$ inside which the different biological parameters (survival, transition, capture, ...) are set in a row, see Choquet, Rouan, and Pradel (2009) for details.

\section{Models under consideration}

We consider the background of multievent models (Pradel, 2005) to handle state uncertainty in analyzing CR data, which are already implemented in E-SURGE. These models belong to the class of Hidden Markov Models (HMM) as the number of states is assume finite (e.g. Cappe et al. (2005)) and consequently to the class of State-Space Models (SSM) (Murphy, 2002). Multievent models are a restriction of SSM in the sense that the number of states and observations are finite which is often the case for CR data.

\subsection{Generalized linear mixed model}

The class of mixed effects models that E-SURGE may consider can be expressed in the form of generalized linear mixed models (GLMM). We consider for both fixed and random effects, two general sets of effect:

- Set of effect 1: time, age, cohort and group effects.

- Set of effect 2: individual effect.

Considering the general form of GLMM $f(\Theta)=X \beta+Z b$ with $\beta$ the vector of fixed effects and $b$ the vector of random effects is computationally demanding because of the dimension of the problem with so many potential effects. Thus, we have implemented the following restricted form of GLMM by constraining separately categories 1 and 2.

$$
f\left(\Theta_{i}\right)=X_{0} \beta_{0}+X_{i} \beta_{1}+\sum_{l=1}^{L} Z_{l} b_{l}+\sum_{l=L+1}^{L+P} Z_{l, i} b_{l, i} i=1, \ldots, N I
$$

where $b_{l} \in \mathbb{R}^{s_{l}}$ and $b_{l, i} \in \mathbb{R}$ are random effects given by

$$
\left\{\begin{array}{l}
b_{l} \sim \mathscr{N}\left(0, \sigma_{l}^{2} \times I_{s_{l}}\right), l=1, \ldots, L, \\
b_{l, i} \sim \mathscr{N}\left(0, \sigma_{l}^{2}\right), l=L+1, \ldots, L+P .
\end{array}\right.
$$

$b_{l}, l=1, \ldots, L$ are random effects associated to the set of effects $1, s_{l}$ is the number of levels of the random effect $l$ ( $s_{l}=G$ for a group random effects), $b_{l, i}, l=L+1, \ldots, L+P$ are individual random effects assuming that individuals are independent. Matrices $X_{i}$ are individual-specific matrices of individual covariates. They are never stored in the computer because of the memory size needed but rather they are computed each time (see appendix A). In the same way, matrices $Z_{l, i}$ contain either 0,1 or values of individual covariates 
and are never stored. Because we assume that individual are independent then matrices of covariance for each random effects are diagonal. We use this property to implement efficient algorithms.

3.2 General expression of the marginal likelihood

Assuming that individuals are independent, the likelihood for fixed effects for the entire set of capture histories is obtained as the product of the probability $P\left(h_{i} \mid \beta\right)$ of the likelihood for each history $h_{i}$

$$
\prod_{h_{i} \in \mathbf{h}} \operatorname{Pr}\left(h_{i} \mid \beta\right)
$$

, see Choquet, Rouan, and Pradel (2009), p 849 for details on multievent models.

For a GLMM like equation (1), we get the marginal likelihood:

$$
L(\beta, \sigma)=\int \prod_{h_{i} \in \mathbf{h}} \operatorname{Pr}\left(h_{i} \mid \beta\right) \omega(\sigma, x) d x .
$$

where $\sigma$ is the vector of variances associated to the random effects and $\omega(\sigma, x)$ is a product of normal densities associated to random effects $b_{l}$ or $b_{l, i}$.

One might think that the dimension of the integral in equation (3) is of the size of the random effects and in most cases the evaluation of $L(\beta, \sigma)$ is numerically intractable. However, we illustrate below two situations for which the computation of equation (3) in E-SURGE can be made by reducing the dimension of the integral.

3.3 Independent and identically distributed random effect for individual

E-SURGE can now handle mixed models with individual random effects only $(L=0)$ :

$$
f\left(\Theta_{i}\right)=X_{0} \beta_{0}+X_{i} \beta_{1}+\sum_{l=1}^{P} Z_{l, i} b_{l, i}
$$

with $b_{l, i}$ in the form of equation (2). There is no limit for the number of random effects that we can build. However for $P>2$ the fitting step may be time consuming.

In this case and similar to Lemenuel-Diot et al. (2005); Gimenez and Choquet (2010), the marginal likelihood (3) is the product of the probabilities of all individual encounter histories. So equation (3) can be rewritten:

$$
L(\beta, \sigma)=\prod_{h \in \mathbf{h}} \int_{\mathbb{R}^{P}} \operatorname{Pr}(h \mid \beta, x) \omega(\sigma, x) d x .
$$

Example 1: Survival varying with individual covariates and random effect. The following model has been used in Gimenez et al. (2006) with a constant survival across time but dependent on an individual covariates $(\mathrm{m})$ and from an individual random effect $\left(b_{i}\right)$.

$$
\operatorname{logit}\left(\phi_{i k}\right)=\beta_{0}+\beta_{1} \mathrm{~m}_{i}+b_{i}, \quad i=1, \ldots, I
$$


where $b_{i} \sim \mathscr{N}\left(0, \sigma_{b}^{2}\right)$, (i.i.d). In equation (6) $P=1$, the computation of the marginal likelihood according to equation (5) leads to the evaluation of several integrals with a single dimension instead of a potentially large dimension according to equation (3).

3.4 Independent and identically distributed random effect for group

E-SURGE can also handle mixed models with group random effects only $(P=0)$ like:

$$
f\left(\Theta_{i}\right)=X_{0} \beta_{0}+X_{i} \beta_{1}+\sum_{l=1}^{L} Z_{l} b_{l}
$$

with $b_{l}$ in the form of equation (2). There is no limit for the number of random effects that we can build. However for $L>2$ the fitting step may be time consuming."

In this case and similar to section 3.3, the marginal likelihood (3) is the product of the marginal probability for each group. We denote $\mathbf{h}_{g}$, the set of encounter histories inside a group $g$ considered together. So equation (3) can be rewritten:

$$
L(\beta, \sigma)=\prod_{g=1}^{G} \int_{\mathbb{R}^{L}}\left(\prod_{h \in \mathbf{h}_{g}} \operatorname{Pr}(h \mid \beta, x)\right) \omega(\sigma, x) d x .
$$

Example 2: We consider a basic model where recapture rates vary with a group random effect.

$$
\operatorname{logit}\left(p_{g}\right)=\beta_{0}+b_{g}, g=1, \ldots, G
$$

where $b_{g} \sim \mathscr{N}\left(0, \sigma_{b}^{2}\right)$, (i.i.d). In this situation $L=1$ so for evaluating the marginal likelihood (8) we have only $G$ one dimensional integrals to evaluate.

\section{Numerical integration}

Integrals in (5) and (8) have no analytical expression so require numerical approximations. After reparameterization, those integrals have the form:

$$
K(y)=\int_{\mathbb{R}^{d}} k(y, x) \exp ^{-x^{t} x} d x_{1} \ldots d x_{d}
$$

where the dimension $d$ depends on the number of random effects. For equation (5), $d=P$, see also Gimenez and Choquet (2010) for the reparameterization in the case of the CJS model with an individual random effect. We will not consider in this paper model with dependence among individuals or groups, some of them are formally described in Cam (2009). Dependences can be accomodated by normal random effects with non diagonal covariance matrices. The marginal likelihood associated to such models can also be expressed as equation (10) after reparameterization, see for example Heiss and Winschel (2008). In the particular case of dependence of individuals and groups, the dimension $d$ of the integral will depend of the structure of the dependence. 
4.1 Order and accuracy

Let $X_{N}$ be a set of $N$ quadrature nodes of $\mathbb{R}^{d}, X_{N}=\left\{x^{(1)}, \ldots, x^{(N)}\right\}$ with $x^{(n)} \in \mathbb{R}^{d}$ for $n=$ $1, \ldots, N$. Let $\Omega_{N}$ be the set of quadrature weights associated with the nodes $X_{N}, \Omega_{N}=$ $\left\{\omega_{1}, \ldots, \omega_{N}\right\}$ with $\omega_{n} \in \mathbb{R}$ for $n=1, \ldots, N$. The integrals $K(y)$ can be approximated by $I I_{N}$ defined by

$$
I I_{N}=\sum_{n=1}^{N} \omega_{n} \times k\left(y, x_{n}\right)
$$

$I I_{N}$ is called a quadrature formula and $K(y)-I I_{N}$ the error functional corresponding to $K(y)$. For relevant sets $X_{N}$ and $\Omega_{N}$, the error functional decreases as $N$ increases. Roughly, two methods of integration exist with different choices for $\Omega_{N}$ and $X_{N}$. The first method is based on the random generation of $X$, see Rosenberg (1967); Genz and Monahan (1999) with or without equidistant properties Zaremba (1968); Gonzalez et al. (2006). Under weak conditions, the simulated value is unbiased and $\sqrt{N}$-consistent by a law of large numbers and independent of the problem. The major drawback of this method relies on the difficulty in determining an appropriate value for $N$ in order to control the error of integration $K(y)-I I_{N}$. The second method is based on the use of quadrature, see Arndt et al. (2006). The Laplace (Liu and Pierce, 1994) method is not considered precise enough for complex CR likelihoods. Consequently, higher orders of Gauss-Hermite quadrature will be considered to obtain better precision. In this case, $N$ increases with $d$ and the order of the quadrature $r$ thus in some cases we can replace the index $N$ by the two indices $d$ and $r$.

For $d=1$, we directly use the Gauss-Hermite quadrature because it adapts easily to the normal density. The nodes of the quadrature $X$ are related to the zeros of the r-th Hermite polynomial with $N=2 \times r-1$. The Gauss-Hermite quadrature with $r$ nodes is exact for polynomials of order $N$. Here as stated in Gimenez and Choquet $(2010), k(y, x)$ is not a polynomial but rather a composite function of a polynomial following an inverse logit function, which can be approximated as closely as long as $N$ is large enough.

Moreover under mild condition (if $\left|K(y)-I I_{d, r+1}\right|<\kappa\left|K(y)-I I_{d, r}\right|, \kappa<1$ ), we can obtain the error estimate associated with a polynomial of order $r$ by considering two successive orders as:

$$
\left|K(y)-I I_{d, r}\right|<\frac{1}{1-\kappa}\left|I I_{d, r+1}-I I_{d, r}\right| .
$$

One severe limitation of Gauss-Hermite quadrature is the effort growing with the dimension of the integral. Multivariate integration formulas are often constructed as a tensor product of quadrature formulas. Let $I I_{1, r}$ be a sequence of quadrature rules on $\mathbb{R}$ then

$$
I I_{d, r}=\sum_{x_{1} \in X} \ldots \sum_{x_{d} \in X} k\left(y, x_{1}, \ldots, x_{d}\right) \prod_{d d=1}^{d} \omega_{d d}\left(x_{d d}\right)
$$

For dimension $d$ and order $r$, a regular grid involves $(M=2 \times r-1)^{d}$ nodes. The calculation of equation (10) very soon becomes intractable. Thus, we try to reduce the order as much as possible with an appropriate scaling.

\subsection{Centering and Scaling}

If the integrand in a numerical integration is not well behaved, algorithms can perform poorly. For one dimension, the order of integration can be set higher to overcome this 
problem. But it is sometimes still not enough, and the error of integration can decrease very slowly. The problem in multiple dimensions is even worse because the number of nodes increases exponentially. Thus for equation (8), we use centering and scaling in a similar way as Lemenuel-Diot et al. (2005) to improve the integration.

In order to base the approximation of the integral (10) on an appropriate range of values of $x$, two transformations are made: centering and scaling. If we assume that $k(y, x)$ is close to a normal distribution of mean $x^{*}$ and variance $S, k(y, x) \times \exp \left(\left(x-x_{*}\right)^{t} S\left(x-x_{*}\right)\right)$ will be close to a constant and the approximation of the integral exact. Thus, we take $x^{*}$ as the mode of $k(y, x)$ and $S$ as half of the inverse of the hessian matrix of the same function.

With the overall transformation $x=U^{-1} \times z+x^{*}$ with $S=U^{t} U$ then equation (10) becomes

$$
\begin{aligned}
K(y) & =\frac{1}{\operatorname{det} U} \int_{\mathbb{R}^{d}} k\left(y, U^{-1} \times z+x^{*}\right) \exp ^{-\left(U^{-1} \times z+x^{*}\right)^{t}\left(U^{-1} \times z+x^{*}\right)} d z_{1} \ldots d z_{d} \\
& =\frac{1}{\operatorname{det} U} \int_{\mathbb{R}^{d}} k_{1}(y, z) \exp ^{-z^{t} \times z} d z_{1} \ldots d z_{d}
\end{aligned}
$$

with $k_{1}(y, z)=k\left(y, U^{-1} \times z+x^{*}\right) \exp ^{-\left(U^{-1} \times z+x^{*}\right)^{t}\left(U^{-1} \times z+x^{*}\right)+z^{t} z}$.

We compute $U$ as in Lemenuel-Diot et al. (2005). To get the inverse of $U$ and when necessary (i.e., when $d>1$ ), we use an appropriate algorithm Xu and Qiao (2008) for the Takagy Factorization (a singular value decomposition of the symmetric matrix $S$ ).

\subsection{Avoiding underflow and overflow}

One particularity is that the approximation $I I_{N}$ is not straightforward to implement in the case of equation (8). In fact, the function $k(y, x)$ is a polynomial of high degree, with parameters belonging to $[0,1]$. Thus very soon, values of the function $k$ will be not representable in finite numerical precision, i.e. $k(y, x)<2^{-128}$. This limitation is called underflow. Thus we reformulate equation (10) to avoid underflow.

One solution to deal with underflow in equation (10) is to consider $\log k(y, x)$ so that the sum of individual contributions is involved rather than the product.

$$
\begin{aligned}
K(y) & =\int_{\mathbb{R}^{d}} k(y, x) \exp ^{-x^{t} x} d x_{1} \ldots d x_{d} \\
& =\int_{\mathbb{R}^{d}} \exp (\log k(y, x)) \exp ^{-x^{t} x} d x_{1} \ldots d x_{d}
\end{aligned}
$$

However, overflow also occurs with $\exp (\log k(y, x))$. One way to deal with this new problem is to scale the quantity $\log k(y, x)$ by an appropriate value called $d e v^{*}$.

$$
\begin{aligned}
K(y) & =\exp \left(0.5 \times d e v^{*}\right) \times \int_{\mathbb{R}^{d}} \exp \left(\log k(y, x)+0.5 \times d e v^{*}\right) \exp ^{-x^{t} x} d x_{1} \ldots d x_{d} \\
& =\exp \left(0.5 \times d e v^{*}\right) \times \int_{\mathbb{R}^{d}} k_{2}(y, x) \exp ^{-x^{t} x} d x_{1} \ldots d x_{d}
\end{aligned}
$$

where $k_{2}(y, x)=\exp \left(\log k(y, x)+0.5 \times d e v^{*}\right)$. 


\section{Implementation in program E-SURGE}

5.1 Details of the global algorithm

To get the MLE, we consider a Quasi-Newton algorithm with a first order difference scheme to approximate the gradient and a relative tolerance of $10^{-6}$. The error made by using a first or second degree finite difference scheme does not influence the result in most of the case (Appendix B). In this paper, the results are obtained with a second-order finite difference scheme although the use of the first order scheme led to the same results in both applications. For a group random effect, we consider the adaptative scaling described previously. To ensure precision of the quadrature scheme, we update the matrix $K$ every 15 iterations of the quasi-Newton algorithm.

\subsection{Description of the random effects}

We extend the model definition language of the tool GEMACO used in M-SURGE and E-SURGE. We refer to Choquet (2008) for the description of the language used for fixed effects.

Although individual covariates are permitted for a fixed effect, individual fixed effects are not allowed in E-SURGE. Therefore, we introduce a new built-in keyword factor denoted IND for individual random effects, and implement random effects for groups with the keyword RANDOM, which translate fixed effects into random effects. These additions fit naturally into E-SURGE's model specification syntax. However contrary to traditional effect like TIME, AGE, GROUP, direct addressing of levels of IND (one level corresponding to one individual) is not currently allowed. We extend also the operator + to concatenate fixed effect and random effect to generate mixed model of the form $(4,7)$. Examples include:

The phrase "I+XIND(1)+IND" models equation (6).

The phrase "I+RANDOM(GROUP)" models equation (9).

More generally, two general forms of phrase are currently allowed

"phrase1+phrase2.IND" for equation (4) and

"phrase1+RANDOM(phrase2)" for equation (7),

where phrase 1 and phrase 2 are any general phrases for fixed effects.

\section{Applications}

\subsection{Application 1}

We consider the European Dipper data used in Lebreton et al. (1992). Although affecting only the two last occasions, we found a trap-dependence effect using program U-CARE (Choquet, Lebreton, Gimenez, Reboulet, and Pradel, 2009). This local effect on time may 
be a consequence on the observation being done on the nest: an individual seen one year on a nest have a greater probability to be detected the next year on the same nest. This effect may be not detected the first years because of the flood years: their livelihood is closely dependant on stream as their nest are close to the water. Therefore, we considered a modified version of the standard Cormack-Jolly-Seber model incorporating a trap-dependence effect to account for this lack of fit. More precisely, we considered two detection probabilities at time $\mathrm{t} p_{t}^{*}$ and $p_{t}$ depending on whether an individual was previously captured or not at the previous occasion $\mathrm{t}-1$.

As in Royle (2008), we tested for individual effects in both survival $(\phi)$ and capture $\left(p^{*}, p\right)$. Similar to Lebreton et al. (1992), the fixed effect for survival is the flood effect $(t=1,4,5,6$ vs $t=2,3)$. With E-SURGE, the sentence for the survival TIME(1 456,23$)+$ IND builds a model where

$$
\begin{aligned}
\operatorname{logit}\left(\phi_{t=(1,4,5,6), i}\right) & =\beta_{1}+b_{1, i}, \\
\operatorname{logit}\left(\phi_{t=(2,3), i}\right) & =\beta_{2}+b_{1, i} .
\end{aligned}
$$

where $b_{1, i} \sim \mathscr{N}\left(0, \sigma_{1}^{2}\right)$.

Using the formulation for trap-dependence described in Gimenez et al. (2003), the set of states used in E-SURGE is \{'Alive and captured previously', 'Alive and not captured previously', 'Dead' $\}$. The sentence for capture TIME(1:4)+FROM.TIME(5 6)+IND builds a model where

$$
\begin{aligned}
\operatorname{logit}\left(p_{t=(1, \ldots, 4), i}^{*}\right) & =\beta_{3}+b_{2, i}, \\
\operatorname{logit}\left(p_{t=(1, \ldots, 4), i}\right) & =\beta_{3}+b_{2, i}, \\
\operatorname{logit}\left(p_{t=(56), i}^{*}\right) & =\beta_{4}+b_{2, i}, \\
\operatorname{logit}\left(p_{t=(56), i}\right) & =\beta_{5}+b_{2, i} .
\end{aligned}
$$

where $b_{2, i} \sim \mathscr{N}\left(0, \sigma_{2}^{2}\right)$.

We fit this model $\phi($ flood $+i n d), p(\operatorname{partial}(m)+i n d)$ using $r=15$ for the quadrature without centering and scaling. In this example, centering and scaling failed to improve the precision of the Gauss-Hermite quadrature because $h(x)$ is not close to a normal distribution. Table (1) shows that no individual effect is detected. For any of the two models $\phi($ flood + ind $), p(\operatorname{partial}(m)+i n d)$ and $\phi($ flood $+i n d), p(m+i n d)$ for the full trap-dependence, the deviance does not even change compared to $\phi($ flood $), p(\operatorname{partial}(m))$ and $\phi($ flood $), p(m)$. In each case, $\sigma_{1}$ and $\sigma_{2}$ are estimated close to zero. Thus, the individual effect detected in Royle (2008) can be explained by unmodeled trap-dependence. This is supported also by simulation where data with trap-dependent effect were generated and then analyzed with an individual effect, the model with individual effect was far better than the constant model (unpublished results). A step-by-step procedure for the implementation of the model in E-SURGE is given in Appendix C.

\subsection{Application 2}

Hantaviruses are the ethiological agents of several more or less severe diseases in humans. In Northern, Western and Central Europe, Puumala hantavirus causes Nephropathia epidemica, a mild form of haemorrhagic fever with renal syndrome Klein and Calisherz (2007). However, for the virus, humans act only as dead-ends and its reservoir host is a small mammal, 


\begin{tabular}{|l|l|l|}
\hline Model & $\mathrm{Np}$ & Deviance \\
$\phi($ flood + ind $), p(\operatorname{partial}(m)+i n d)$ & 7 & 650.910 \\
$\phi($ flood $), p(\operatorname{partial}(m)+i n d)$ & 6 & 650.910 \\
$\phi($ flood + ind $), p($ partial $(m))$ & 6 & 650.910 \\
$\phi($ flood $), p($ partial $(m))$ & 5 & 650.910 \\
$\phi($ flood + ind $), p(m+$ ind $)$ & 6 & 656.225 \\
$\phi($ flood $), p(m)$ & 4 & 656.225 \\
$\phi($ flood $), p$ & 3 & 660.102 \\
\hline
\end{tabular}

Table 1 European Dipper example revisited. $N p$ is the number of mathematical parameters of the model.

\begin{tabular}{|l|l|l|}
\hline Model & $\mathrm{Np}$ & Deviance \\
$\phi(i), p(i+r(g))$ & 3 & 944.013 \\
$\phi(i), p(i)$ & 2 & 947.381 \\
\hline
\end{tabular}

Table 2 Bank Vole example with a random effect on group. $i$ is for the intercept, $r(g)$ is for a group random effect. $N p$ is the number of mathematical parameters of the model.

the Bank Vole, Myodes glareolus. To better understand the epidemics in humans, it is crucial to understand the dynamics of Puumala virus within the reservoir host populations. The data were collected to investigate the impact of Puumala virus on the demography of Bank Voles. Nine sites, typical for the optimal bank vole's habitat, were monitored in the endemic zone where most human cases have occurred in the last years in Belgium. In each site, six trapping sessions were carried out from 2004 to 2006 with 100 live traps in a 1 ha grid Tersago (shed). Sites are coded as nine groups which are in fact geographic sites without movement among sites. Because the purpose here is not a full epidemiological study, we consider only two states \{'Alive','Dead'\} and we construct a simple survival model with coupled random group effects on capture. The sentence for capture (decomposed in E-SURGE as first capture $=$ FIRSTE and recapture $=$ NEXTE $)$ FIRSTE + NEXTE + RANDOM (NEXTE.GROUP) builds a model where

$$
\operatorname{logit}\left(p_{\text {age }=2, g}\right)=\beta_{2}+b_{g} .
$$

where $b_{g} \sim \mathscr{N}\left(0, \sigma^{2}\right)$ and the first mathematical parameter(FIRSTE) is fixed to 1 in the biological scale $\left(p_{\text {age }=1}=1\right)$. Note that this approach is equivalent to conditioning at first capture.

We fit the model using $r=15$ with centering and scaling. A step by step procedure for the implementation of the model in E-SURGE is given in Appendix D. Results are represented in Table 2. The LRT test between models $\phi(i), p(i+r(g))$ and $\phi(i), p(i)$ was accepted/rejected.

\section{Discussion}

We have implemented effective algorithms in E-SURGE to fit models and get maximum likelihood parameter estimates with random effects in cases where independence of individuals or groups is assumed. Instead of a MCMC approach, we consider a Gauss-Hermite scheme to cope with high-dimensional integrals. This approach has several advantages over Monte-Carlo approaches. For example, we obtained a cheap measure of the error. A better measure could be achieved for both methods by computing the discrepancy (Frank and Heinrich, 1996), but this option comes at a price. Furthermore, the language inside GEMACO has been extended to allow built-in mixed models incorporating individual and 
group random effects with i.i.d. assumptions. Thus, practitioners without experience in MCMC modeling can access these modeling tools within the scope of a familiar, userfriendly software package.

However several challenges remain:

- First, built-in time, age and cohort random effects still need to be implemented in E-SURGE. Dimensions of the associated integrals are very high and additional algorithms must be implemented to fit these models in a reasonable amount of time. Much progress have been done the past few years with MCMC methods or EMtype algorithms (Chaubert-Pereira, 2008). Our ultimate goal is to implement the most appropriate algorithm for each model.

- Second, in the present version of E-SURGE, the independence of groups needs to be specified in a menu of E-SURGE. Description or automatic detection of independence between groups remains to be implemented.

- Third, models with non-diagonal variance-covariance matrices as in Pinheiro and Bates (1996) have to be implemented. The description of dependence between individuals (which would allow fitting animal models like in Meyer (2001)) or groups is the first step of this implementation.

- Fourth, besides fitting the models, model selection remains a challenging exercise with AIC a subject of controversy in the context of mixed models. Other measures like CAIC (Vaida and Blanchard, 2005) should be investigated.

- Fifth, the acute computation of the rank of a mixed model for CR remains to be done to investigate if redundant parameters are present in the models. In the current version of E-SURGE, the rank is set equal to the rank of the hessian of the likelihood.

- Last but not least, goodness-of-fit procedures are missing for CR mixed models. We hope that further progress will be made with GOF as an inappropriate random effect can be selected even with marginal overdispersion. This is the case in application 1.

\section{Acknowledgements}

The authors warmly thank Laurent Crespin for suggesting Application 2 and Tersago Katrien for providing the bank vole (Myodes glareolus) data and two anonymous referees for very useful comments that improved the presentation of the paper. This research was supported by a grant from the 'Jeunes Chercheuses et Jeunes Chercheurs' program of the French ANR (ANR-08-JCJC-0028-01).

\section{Supplementary Materials}

The program E-SURGE is available from http://www.cefe.cnrs.fr/BIOM/logiciels.htm

\section{References}

Arndt, C., Kozlitina, J., and Preckel, P. V. (2006). Efficient survey sampling of households via gaussian quadrature. Journal of the Royal Statistical Society Series C-Applied Statistics 55, 355-364. 
Burnham, K. and Anderson, D. (2002). Model selection and Inference: A practical Information-theoretic approach. New York, 2nd edition.

Cam, E. (2009). Contribution of capture-mark-recapture modeling to studies of evolution by natural selection. In Thomson, D. L., Cooch, E. G., and Conroy, M. J., editors, Modeling Demographic Processes in Marked Populations, volume 3 of Environmental and Ecological Statistics, pages 83-129. Springer.

Cappe, O., Moulines, E., and Ryden, T. (2005). Inference in Hidden Markov models. Springer series in Statistics. Springer.

Chaubert-Pereira, F. (2008). Combinaisons Markoviennes et semi-Markoviennes de modeles de regression. Application a la croissance d'arbres forestiers. $\mathrm{PhD}$ thesis.

Choquet, R. (2008). Automatic generation of multistate capture-recapture models. The Canadian Journal of Statistics 36, 43-57.

Choquet, R., Lebreton, J. D., Gimenez, O., Reboulet, A., and Pradel, R. (2009). U-CARE: Utilities for performing goodness of fit tests and manipulating CApture-REcapture data. Ecography 32, 1071-1074.

Choquet, R., Reboulet, A., Pradel, R., Gimenez, O., and Lebreton, J. D. (2004). M-SURGE: New software specifically designed for multistate recapture models. Animal Biodiversity and Conservation 27, 207-215.

Choquet, R., Rouan, L., and Pradel, R. (2009). Program E-SURGE: A software application for fitting multievent models. In Thomson, D. L., Cooch, E. G., and Conroy, M. J., editors, Modeling Demographic Processes in Marked Populations, volume 3 of Springer Series: Environmental and Ecological StatisticsEnvironmental and Ecological Statistics, pages 845-865, Dunedin. Springer.

Clark, J. S. (2005). Why environmental scientists are becoming bayesians. Ecology Letters 8, 2-14.

Coull, B. A. and Agresti, A. (1999). The use of mixed logit models to reflect heterogeneity in capture-recapture studies. Biometrics 55, 294-301. ISI Document Delivery No.: 183RK Times Cited: 56 Cited Reference Count: 21 INTERNATIONAL BIOMETRIC SOC.

Dennis, J. and Schnabel, R. (1983). Numerical Methods for Unconstrained Optimization and Nonlinear Equations. Classics in applied mathematics. SIAM.

Frank, K. and Heinrich, S. (1996). Computing discrepancies of smolyak quadrature rules. Journal of Complexity 12, 287-314.

Genz, A. and Monahan, J. (1999). A stochastic algorithm for high-dimensional integrals over unbounded regions with gaussian weight. Journal of Computational and Applied Mathematics 112, 71-81.

Gimenez, O., Bonner, S., King, R., Parker, R. A., Brooks, S. P., Jamieson, L., Grosbois, V., Morgan, B., and Thomas, L. (2009). Winbugs for population ecologists: Bayesian modeling using markov chain monte carlo methods. In Thomson, D., Cooch, E., and Conroy, M., editors, Modeling Demographic Processes in Marked Populations, volume 3 of Springer Series: Environmental and Ecological StatisticsEnvironmental and Ecological Statistics, pages 883-915, Dunedin. Springer.

Gimenez, O. and Choquet, R. (2010). Incorporating individual heterogeneity in capturerecapture models using numerical integration. Ecology 91, 148-154.

Gimenez, O., Choquet, R., and Lebreton, J. D. (2003). Parameter redundancy in multistate capture-recapture models. Biometrical Journal 45, 704-722.

Gimenez, O., Covas, R., Brown, C., Anderson, M., Brown, M., and Lenormand, T. (2006). Nonparametric estimation of natural selection on a quantitative trait using mark-recapture data. Evolution 60, 460-466. 
Gonzalez, J., Tuerlinckx, F., De Boeck, P., and Cools, R. (2006). Numerical integration in logistic-normal models. Computational Statistics and Data Analysis 51, 1535-1548.

Heiss, F. and Winschel, V. (2008). Likelihood approximation by numerical integration on sparse grids. Journal of Econometrics 144, 62-80.

Klein, S. L. and Calisherz, C. H. (2007). Emergence and persistence of Hantaviruses, volume 315 of Wildlife and Emerging Zoonotic Diseases: The Biology, Circumstances and Consequences of Cross-Species Transmission. Springer-Verlag Berlin, Berlin.

Lebreton, J.-D., Burnham, K., Clobert, J., and Anderson, D. (1992). Modeling survival and testing biological hypotheses using marked animals: A unified approach with case studies. Ecological Monographs 62, 67-118.

Lemenuel-Diot, A., Mallet, A., Laveille, C., and Bruno, R. (2005). Estimating heterogeneity in random effects models for longitudinal data. Biometrical Journal 47, 329-345.

Link, W. A. and Barker, R. J. (2005). Modeling association among demographic parameters in analysis of open population capture-recapture data. Biometrics 61, 46-54.

Liu, Q. and Pierce, D. (1994). A note on gauss-hermite quadrature. Biometrika 81, 624-629.

Lunn, D., Thomas, A., Best, N., and Spiegelhalter, D. (2000). Winbugs a bayesian modelling framework: Concepts, structure, and extensibility. Statistics and computing 10, 325-337.

McClintock, B. T., White, G. C., Antolin, M. F., and Tripp, D. W. (2009). Estimating abundance using mark-resight when sampling is with replacement or the number of marked individuals is unknown. Biometrics 65, 237-246.

Meyer, K. (2001). Estimating genetic covariance functions assuming a parametric correlation structure for environmental effects. Genetics Selection Evolution 33, 557585 .

Murphy, K. (2002). Dynamic Bayesian Networks: Representation, Inference and Learning. $\mathrm{PhD}$ thesis.

Pinheiro, J. C. and Bates, D. M. (1996). Unconstrained parametrizations for variancecovariance matrices. Statistics and computing 6, 289-296.

Pradel, R. (2005). Multievent: An extension of multistate capture-recapture models to uncertain states. Biometrics 61, 442-447.

Rosenberg, 1. (1967). Bernstein polynomials and monte-carlo integration. SIAM J. Numer. Anal. 4, 566-574.

Royle, J. A. (2008). Modeling individual effects in the cormack-jolly-seber model: A statespace formulation. Biometrics 64, 364-370.

Royle, J. A. and Link, W. A. (2002). Random effects and shrinkage estimation in capturerecapture models. Journal of Applied Statistics 29, 329-351. ISI Document Delivery No.: 511CT Times Cited: 5 Cited Reference Count: 38 CARFAX PUBLISHING.

Tersago, K. (Unpublished). Spatio-temporal variation of Puumala hantavirus in Belgium: en eco-epidemiological study. $\mathrm{PhD}$ thesis.

Vaida, F. and Blanchard, S. (2005). Conditional akaike information for mixed-effects models. Biometrika 92, 351-370.

Wintrebert, C. M. A., Zwinderman, A. H., Cam, E., Pradel, R., and van Houwelingen, J. C. (2005). Joint modelling of breeding and survival in the kittiwake using frailty models. Ecological Modelling 181, 203-213.

$\mathrm{Xu}$, W. and Qiao, S. Z. (2008). A divide-and-conquer method for the takagi factorization. Siam Journal on Matrix Analysis and Applications 30, 142-153.

Zaremba, S. K. (1968). Mathematical basis of monte carlo and quasi-monte carlo methods. Siam Review 10, 303-314. 
A Implementation of the structure of $X_{i}$ and $Z_{l, i}$

$X_{i}=A \times B_{i}$ where $B_{i}$ is set of square matrices dependent on individuals. This structure allows us to consider model like: I+T.XIND. In this case, $A$ is a time dependent matrix and the matrix $B_{i}$ is a $(K-1) \times(K-1)$ diagonal matrix which entries are the value of the covariates for individual $i$.

$Z_{l, i}=C_{l} \times D_{l, i}$ where $D_{1, i}$ is a square matrix depending of the individual and of the effect. This structure allows us to consider model like: I+XIND+RAND(XIND). In this case $L=0, P=1, C_{1}$ is a constant vector of 1 and $D_{1, i}$ is the value of the covariates for individual $i$.

\section{B Influence of the finite difference scheme}

We check that the tolerance $\left(\mathrm{Tol}=10^{-6}\right.$ ) used for the quasi-Newton algorithm on the gradient and the error $(E r r)$ made by the approximation of the gradient using a finite difference scheme and a numerical integration are consistant, i.e. of the same order. Let $\varepsilon$ be the computer precision, we will demonstrate that the global error $E r r$ defined by:

$$
E r r=\left.\frac{\partial K(y)}{\partial y}\right|_{y_{0}}-\sum_{n=1}^{N} \omega_{n} \times \frac{k\left(y_{0}+\sqrt{\varepsilon}, x_{n}\right)-k\left(y_{0}, x_{n}\right)}{\sqrt{\varepsilon}}
$$

can be decomposed as a sum of two sources of error; the error of the finite difference scheme applied to the gradient of $g$ and the error made by the quadrature formulae applied to the gradient of $g$. By the Fubini theorem,

$$
\nabla K\left(y_{0}\right)=\int_{\mathbb{R}^{d}} \nabla_{y} k\left(y_{0}, x\right) \exp ^{-x^{t} x} d x_{1} \ldots d x_{d}
$$

The error made by approximating a derivatives by a first order finite-difference scheme is $O(\sqrt{\varepsilon})$ (see Dennis and Schnabel (1983)) so

$$
\begin{aligned}
& E r r=\nabla K\left(y_{0}\right)-\sum_{n=1}^{N} \omega_{n} \times\left(\nabla_{y} k\left(y_{0}, x_{n}\right)+O(\sqrt{\varepsilon})\right) \\
& E r r=\int_{\mathbb{R}^{d}} \nabla_{y} k\left(y_{0}, x\right) \exp ^{-x^{t} x} d x_{1} \ldots d x_{d}-\sum_{n=1}^{N} \omega_{n} \times \nabla_{y} k\left(y_{0}, x_{n}\right)+O(\sqrt{\varepsilon})
\end{aligned}
$$

For Matlab on a 32 bits windows system, $\varepsilon=2.2204 \times 10^{-16}$ so as soon as the error made by the quadrature formulae is lower than $\mathrm{Tol}$, the global error is lower than $\mathrm{Tol}$. For a lower tolerance $\left(\mathrm{Tol}=10^{-6}\right)$ used for the quasi-Newton algorithm on the gradient then a second-order finite-difference scheme should be used.

\section{Application 1 with E-SURGE}

E-SURGE can accept capture-recapture data either in MARK or BIOMECO format. The two types of data file are not very different: each row corresponds to a particular capture history followed by the number of individuals with that history. In MARK format this count is followed by a semi-colon and in BIOMECO format the data are preceded by the number of different capture histories and the number. In this study, there is only one site. We will fit here the model $\phi($ flood $) p(\operatorname{partial}(m)+i n d)$ described in part 6.1.

\section{C.1 Starting E-SURGE}

From E-SURGE, start a new session named 'result.mod'. Read in the data file and tell to E-SURGE that there is no individuals covariates. Check that the numbers of capture occasions, groups and events are correct (in this case 7 capture occasions, 1 group and 2 events): E-SURGE makes assumptions about the number of states, but these need to be modified depending on the problem you have to treat. In this case, we change the number of states to three. We will also have to set the number of age classes to one as for the present, we won't consider any age effect. 
C.2 Fitting the model

Press the Modify button, and change the settings to specify that there a single age class, a single group and 3 states (see Fig. 1).

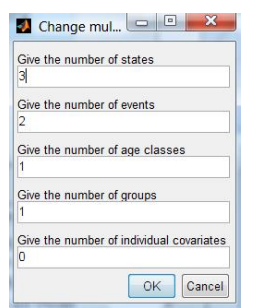

Fig. 1 E-SURGE: The number of states is set to 3 and the number of age classes is set to one.

The main menu should show the changes. Fitting the models in E-SURGE involves four steps:

1. The Gepat step: specifying which ones of all the potential parameters have to be estimated, which ones will be calculated as the complement to 1 (there is one such parameter per multinomial) and which ones correspond to impossible events or transitions and are fixed to zero;

2. The Gemaco step: specifying the effects (time, age) acting on the active parameters;

3. The IVFV step: specifying initial values for the optimization procedure and/or fixed values for the active parameters;

4. The RUN step: launching the optimization procedure.

\section{C.2.1 Specifying the pattern matrices using the GEPAT interface}

There are three types of parameters used in the definition of a multi-event model (Pradel 2005):

1. the initial state probabilities;

2. the transition probabilities;

3. the event probabilities.

Each type of parameters is gathered into a row-stochastic matrix, i.e. each row corresponds to a multinomial. (Each matrix can be further decomposed into a product of several stochastic matrices allowing for example to estimate separately survival and transition parameters. However, for the current model, only 1 step is required for each type of matrix.)

To enter the GEPAT interface, click the GEPAT button at the bottom of the main window. The GEPAT interface screen for the initial state pattern matrix appears.

By default, E-SURGE let all live states available as initial steps. The state dead, last in the list, is impossible and does not even appear. The last live step is taken by default to be the one whose probability will be calculated indirectly, as the complement of those of the other live states. This is specified in the above window using the following general conventions for Gepat:

- a minus sign (-) indicates that a potential parameter is unavailable in the current model (impossible transition for instance). This is equivalent to fixing it to zero but more explicit;

- any greek letter (strike a latin letter and E-SURGE will show its greek counterpart) indicates a free parameter, one to be estimated directly. Note that the particular greek letter entered is totally irrelevant to E-SURGE. In particular, the same greek letter is used repeatedly by default within a pattern matrix ( by default for initial states); this does NOT mean that the parameters are being forced to be equal;

- the asterisk (*) indicates the parameter that is calculated indirectly, as the complement to 1 of all the other parameters on the same row. There MUST always be one and only one asterisk per row because each row corresponds to a multinomial. 


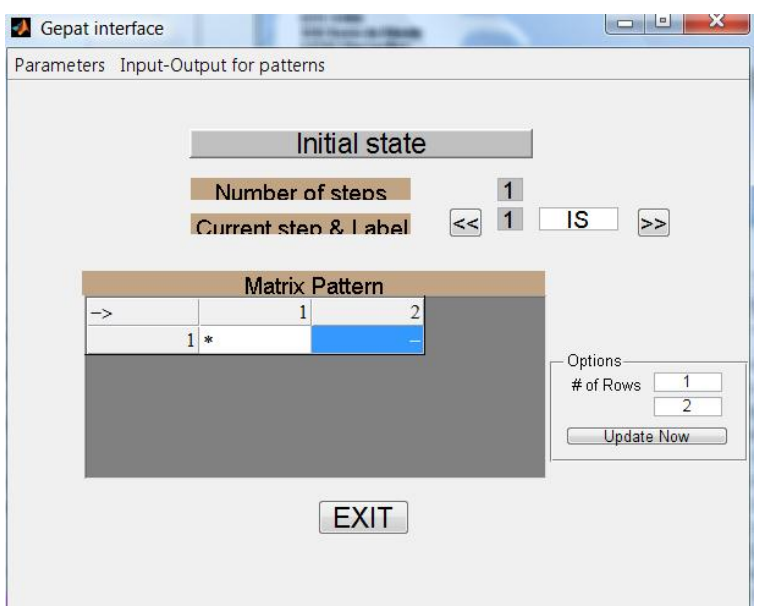

Fig. 2 E-SURGE: Pattern for the intial states vector.

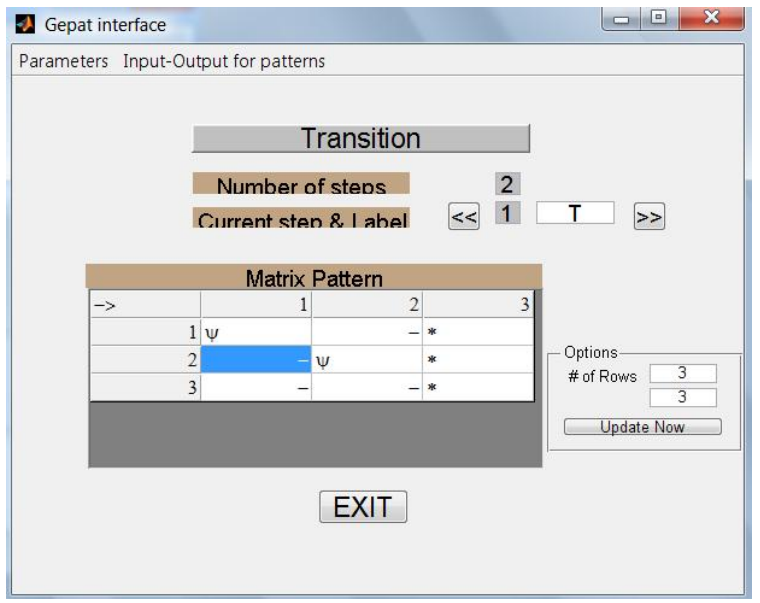

Fig. 3 E-SURGE: Pattern for the survival matrix.

Note that the order of the states is chosen by the user except for the dead state that is always positioned last. Here, the default pattern is not correct for transition and event. We need to change them.

For intial states, we implement the pattern given in Figure 2. For transitions, we need two steps, one for survival and one for capture. So we set the number of steps to 2. For survival, we implement the pattern given in Figure 3. For capture, we implement the pattern given in Figure 4. For event, we implement the pattern given in Figure 5. Press the "EXIT" button to return to the main window.

\section{C.2.2 Specifying the model using the GEMACO interface}

The GEMACO interface uses keywords to create a modelling sentence that indicates how parameters vary by time, over groups, over age classes, etc. At the end of the GEMACO procedure, a design matrix is created for each type of parameters. Each row of the design matrix will correspond to a parameter of the full model (all potential variability: time, age, group) and each column corresponds to a parameter of the actual model.

The GEMACO syntax is fairly intuitive but the "sentences" you enter in the GEMACO interface must respect 


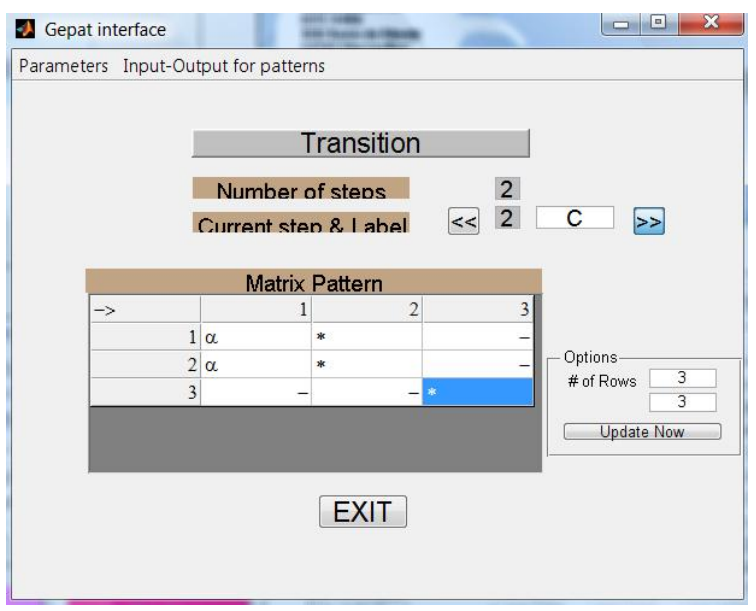

Fig. 4 E-SURGE: Pattern for the capture matrix for the trap-dependent model.

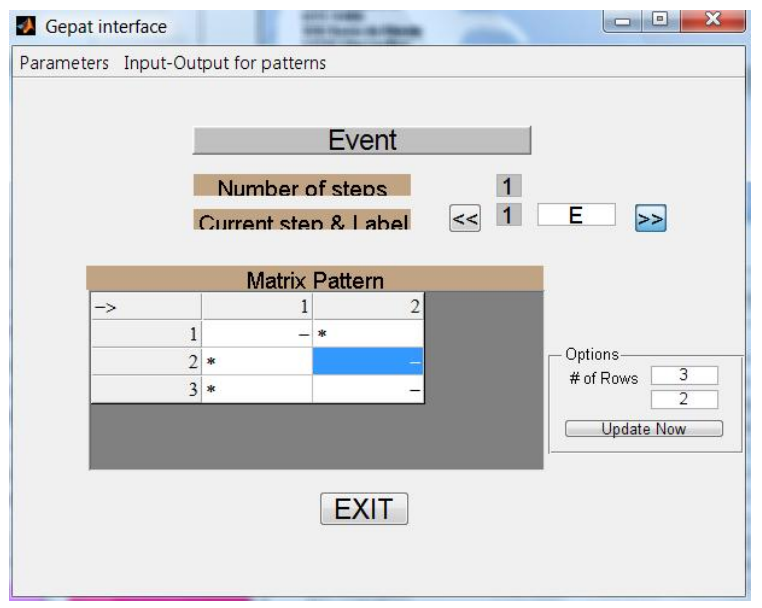

Fig. 5 E-SURGE: Pattern for the event matrix.

some priority rules that we will not develop here. We encourage the user to read the E-SURGE user manual and the paper (Choquet, 2008) in which the GEMACO syntax is fully explained.

In this example, we only want to show how to use E-SURGE to fit our model.

For the trap-dependent model, the set of initial state probabilities and the set of event probabilites are empty. Click on the top "Initial State" button to go to the "Transition" screen.

The survival and the capture modelling

To specify the model on survival, we use the phrase $\mathrm{T}(1456,23)$ for the flood effect, the new keyword IND for the individual random effect. As we combine all, the GEMACO sentence becomed T(1 456,23$)+$ IND. Select the next step for transitions which corresponds to the modelling of trap dependence. We use the phrase $\mathrm{T}(1: 4)+\mathrm{T}(5$ 6) $. \mathrm{F}+\mathrm{IND}$.

At this stage, to create the design matrices, we click on the Gemaco item in the top menu and select the "call GEMACO (all phrases)" submenu. At this stage, all the model structures are specified and the design 
matrices appear in the left window of each screen of the GEMACO interface; press the "EXIT" button to return to the main window.

\section{C.2.3 Specifying the initial and fixed values using the IVFV interface}

In E-SURGE, the user can choose the way to generate the initial values of the optimization procedure. They can be either "constant", "randomly generated" or "equal to the estimates of a previously fitted model". Once the type of initial values is chosen, the user can also fix the values for some parameters using the IVFV interface. Press the IVFV button to enter the interface (Fig. 6). As there is no need to specify neither fixed values nor initial values. Click on the "EXIT" button to return to the main window.

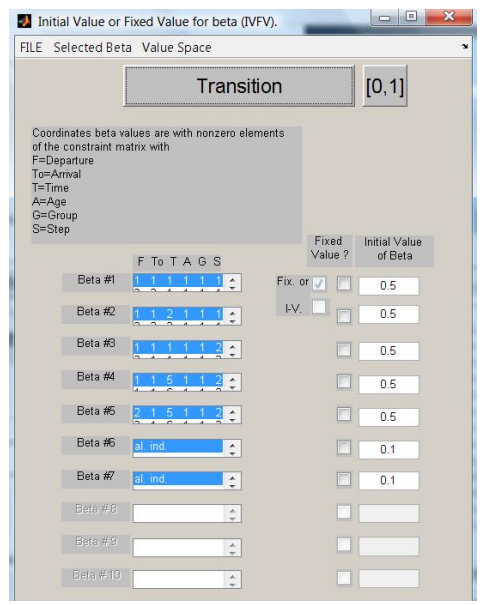

Fig. 6 E-SURGE: The initial values fixed values interface. The two last parameters are the parameter associated to the two random effects.

\section{C.2.4 Running the model}

Before running the model, we have to specified the method of integration, here we choose the classical GaussHermite method (set by default) described in the paper with 29 quadrature nodes ( $\mathrm{r}=15$, set by default). We also tick the "compute C-I(Hessian)" option to get confidence intervals. The model is now ready to be fitted to the data. Press the RUN button. We observe in Figure 7 that at the end of the fit, the estimate error made by the GH scheme to get the likelihood is lower than $10^{-} 6$ as $-15.64 \leq-6$.

In Figure 8, we get the estimates for the model $\phi(\operatorname{flood}+i n d) p(\operatorname{partial}(m)+i n d)$ in the mathematical scale.

\section{Application 2 with E-SURGE}

We will fit here the model $\phi($ flood $) p(\operatorname{partial}(m)+i n d)$ described in part 6.2 with E-SURGE.

\section{D.1 Starting E-SURGE}

From E-SURGE, start a new session and load the data set. For this application, we only need to change the number of age classes to one as for the present, we won't consider any age effect. 


\begin{tabular}{|c|c|c|c|c|}
\hline 1 & 126 & b 1.14 & 1.12186 & -U.uบUชss \\
\hline 8 & 145 & 650.978 & 4.71931 & $9.37 e-005$ \\
\hline 9 & 163 & 650.933 & 1.23147 & $-3.58 e-006$ \\
\hline 10 & 181 & 650.917 & 1.32543 & $-4.34 e-005$ \\
\hline 11 & 200 & 650.911 & 2.33857 & $-2.65 e-005$ \\
\hline 12 & 218 & 650.91 & 1.65152 & $-1.34 \mathrm{e}-005$ \\
\hline 13 & 236 & 650.91 & 0.954357 & $-1.12 e-006$ \\
\hline 14 & 254 & 650.91 & 0.791328 & $-3.81 e-007$ \\
\hline 15 & 272 & 650.91 & 0.923187 & $-1.86 e-007$ \\
\hline 16 & 290 & 650.91 & 1 & $2.89 e-008$ \\
\hline 17 & 309 & 650.91 & 1.62486 & $-2.57 e-009$ \\
\hline 18 & 328 & 650.91 & 15.3682 & $-1.02 e-009$ \\
\hline 19 & 346 & 650.91 & 1.86922 & $-1.38 e-008$ \\
\hline 20 & 364 & 650.91 & 1.97872 & $8.8 \mathrm{e}-010$ \\
\hline \multicolumn{5}{|c|}{$\begin{array}{l}\text { Optimization terminated successfully: } \\
\text { Current search direction is a descent direction, and magnitude of } \\
\text { directional derivative in search direction less than } 2 \text { *options.TolFu } \\
\text { log10 of relative estimate error in integration: order } 16-15=-14.6439\end{array}$} \\
\hline
\end{tabular}

Fig. 7 E-SURGE: The Output during the RUN step shows that 20 iterations are needed for convergence and that the error due to numerical integration is small (lower than $10^{-} 6$ ).

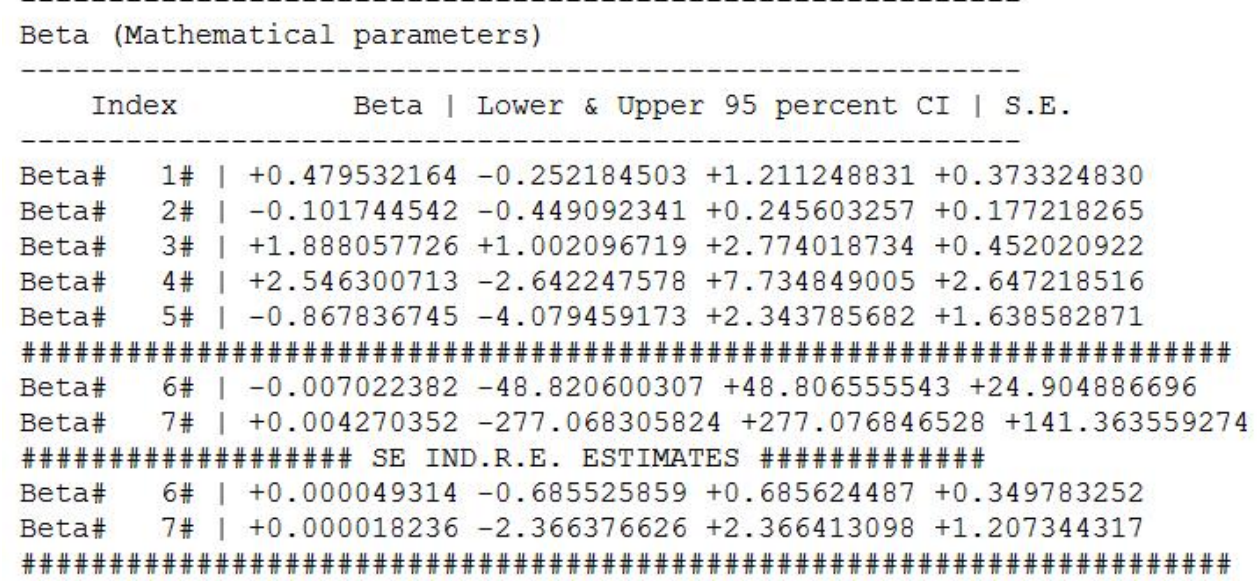

Fig. 8 E-SURGE: Estimates and standard errors for the model $\phi($ flood $+i n d) p(\operatorname{partial}(m)+i n d)$.

D.2 Fitting the model

Press the Modify button, and change the settings to specify that there is a single age class, nine groups and 2 states (see Fig. 9).

The main menu should show the changes. Fitting the models in E-SURGE involves four steps:

\section{D.2.1 Specifying the pattern matrices using the GEPAT interface}

Note that the order of the states is chosen by the user except for the dead state always positioned last. Here, the default pattern is one of the CJS model. We don't need to change them. 


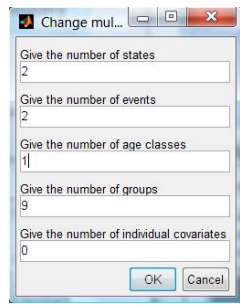

Fig. 9 E-SURGE: The number of age classes is set to one.

\section{D.2.2 Specifying the model using the GEMACO interface}

In this example, we only want to show how to use E-SURGE to fit our model.

The initial states modelling

For the CJS model, the set of initial state probabilities is empty. Click on the top "Initial State" button to go to the "Transition" screen.

The survival and the capture modelling

To specify the model on survival, we use the phrase I (see Fig. 10).

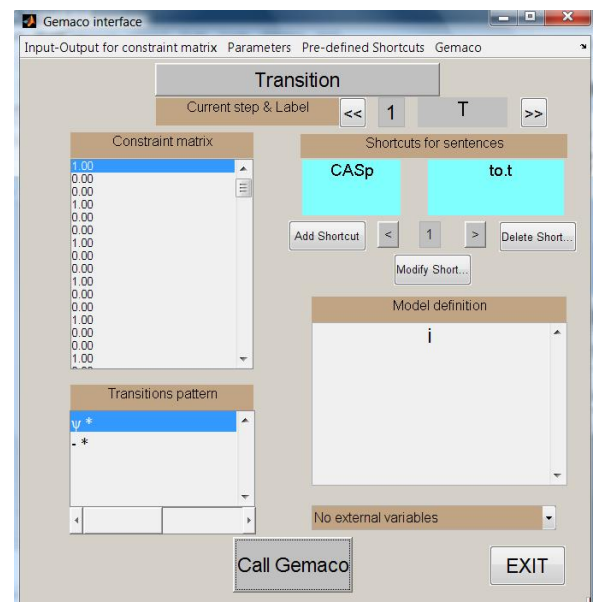

Fig. 10 E-SURGE: The sentence 'i' builds the model $\phi($.$) .$

Click on the top "Transition" button to go to the "Event" screen.

The event modelling

Because the model conditions on the first capture occasion of each individual, the only event to model at the time of the first encounter is the site of capture. It is only later on that the event 'not encountered' becomes possible. Thus, the event probabilities at the time of the first encounter must be treated separately. This is achieved through the use of the keywords "firste", which stands for 'first encounter', and "nexte", which stands for 'next encounters', respectively.

The output of Gepat is shown in the 'transitions pattern' subwindow at the bottom left. For each state (in 
row), there is only one active event which is the capture on the relevant site (where the stands), and the other possible event taken as the complement is 'not encountered' (first column). For instance, for state 11, the first row, the individual can be encountered on site 1 (second column, probability to be estimated) or 'not encountered' (first column, probability calculated as 1 - the other probability). The active parameters are thus just the capture probabilities. At the time of the first encounter, the capture is certain and the capture probabilities will all be 1 . At this stage, we cannot specify a fixed value, but we can specify that we need just one parameter common to all states by keeping "firste" by itself. Later, capture probability will be constant. Thus, the complete sentence is "firste+nexte+random(nexte.g)".

At this stage, to create the design matrices, we click on the Gemaco item in the top menu and select the "call GEMACO (all phrases)" submenu (see Fig. 11). All the model structures are now specified and the design matrices for fixed effect appear in the left window of each screen of the GEMACO interface; press the "EXIT" button to return to the main window.

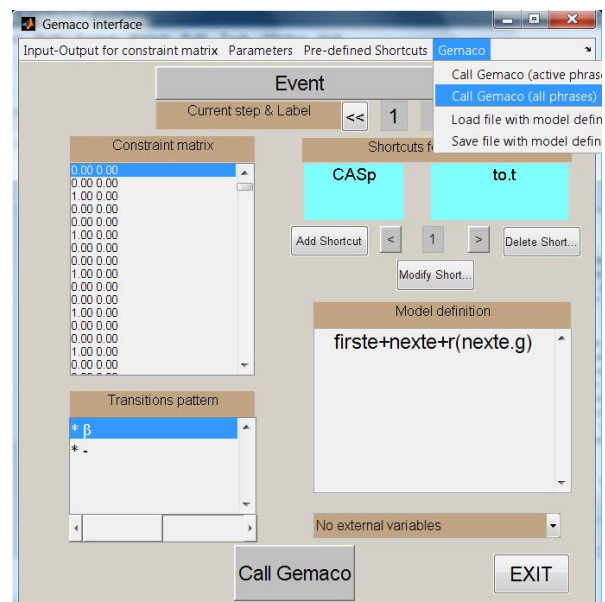

Fig. 11 E-SURGE: The sentence 'firste+nexte+r(nexte.g)' builds the model $\phi(i+r(g))$.

\section{D.2.3 Specifying the initial and fixed values using the IVFV interface}

In E-SURGE, the user can choose the way to generate the initial values of the optimization procedure. They can be either "constant", "randomly generated" or "equal to the estimates of a previously fitted model". Once the type of initial values is chosen, the user can also fix the values for some parameters using the IVFV interface. Press the IVFV button to enter the interface.

The initial states probabilities

In this case, there is no need to specify neither fixed values nor initial values. Click on the top "Initial State" button to arrive at the "Transition" screen.

The survival-transitions probabilities

There is no need to fix values for the transition probabilities so this screen can be left in its default state. Click on the top "Transition" button to arrive at the "Event" screen.

The event probabilities

We can see here the different capture rate appearing in the definition of the model. The series of number indicate successively: 
- the line in the event matrix (corresponding to the state),

- the column in the event matrix (corresponding to the event),

- the capture occasion,

- the age class,

- the group,

- the step in the matrix decomposition of the event matrix(here 1)

Thus, the first parameter corresponds to the capture rate at the first capture occasion for the first age class, i.e. time of first encounter $(A=1)$. This is the only parameter with $A=1$ because we have gathered all the capture rates relative to the first encounter into a single parameter. This parameter needs to be fixed to 1. We do this by entering the value 1 as "Initial Value" and ticking the box nearby (see Fig. 12). The other

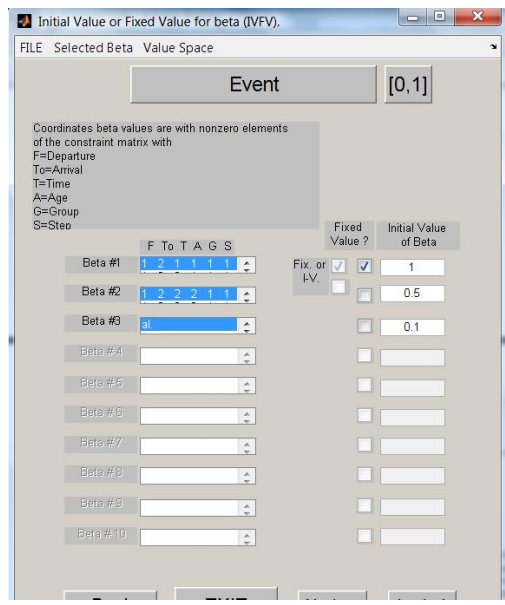

Fig. 12 E-SURGE: The first mathematical parameter corresponding to 'firste' is fixed to 1 by ticking the box nearby. The third mathematical parameter is the starting value for the square root of the standard error, it must be strictly positive.

parameter correspond to the following capture rates $(A=2)$; there is no need to fix these parameters.

After all the fixed values have been specified, press the EXIT button.

\section{D.2.4 Running the model}

Before running the model, we have to specify the method of integration. Click on the button 'Advanced Numerical Options \& Modify' in the main window of E-SURGE. Here we choose the Adaptative GaussHermite method described in the paper (the fourth value is set to 1) with 29 quadrature nodes (the fifth value is set to 15) (see Fig. 13). We also tick the submenu 'Random Effects for Independent Group Only' in the menu 'Models' (Fig. 14). We also tick the 'compute C-I(Hessian)' option to get confidence intervals and an estimated of the model rank. The model is now ready to be fitted to the data. Press the RUN button. We observe in Figure 15 that at the end of the fit, the estimate error made by the GH scheme to get the likelihood is lower than $10^{-} 6$ as $-6.45 \leq-6$.

In Figure 16, we get the estimates for the model $\phi() p.(i+r(g))$ in the mathematical scale. 


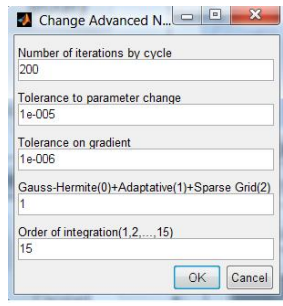

Fig. 13 E-SURGE: We choose the Adaptative Gauss-Hermite method (the fourth value is set to 1) with 15 quadrature nodes (the fifth value is set to 15 ).

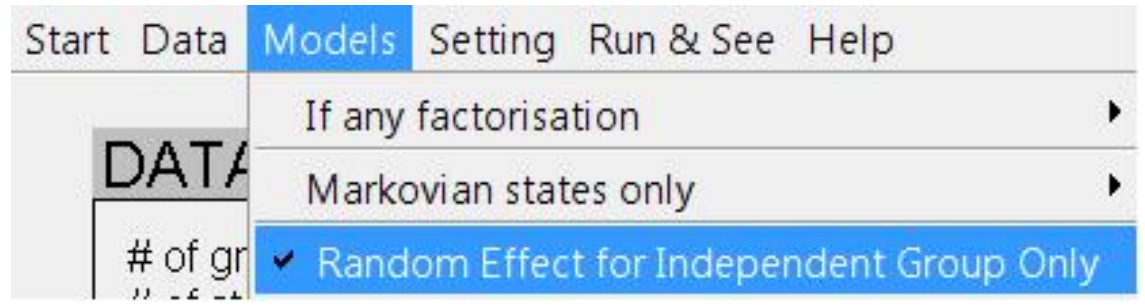

Fig. 14 E-SURGE: To speed-up calculations and improve the precision of the integration, we set that the random group effect is i.i.d.

$\begin{array}{cccrr}\text { Iteration } & \text { Func-count } & \mathrm{f}(\mathrm{x}) & \text { Step-size } & \begin{array}{r}\text { Directional } \\ \text { derivative } \\ 1\end{array} \\ 2 & 16 & 1159.5 & 0.0124954 & -1.86 \mathrm{e}+005 \\ 3 & 27 & 951.553 & 0.00243892 & 2.14 \mathrm{e}+003 \\ 4 & 41 & 947.522 & 0.0456034 & -4.09 \\ 5 & 51 & 944.493 & 0.895395 & 2.31 \\ 6 & 62 & 944.167 & 0.118707 & 0.0121 \\ 7 & 72 & 944.017 & 1.57906 & -0.000216 \\ 8 & 82 & 944.013 & 0.838651 & -5.68 \mathrm{e}-006 \\ 9 & 92 & 944.013 & 1.04738 & -1.6 \mathrm{e}-005 \\ 10 & 102 & 944.013 & 0.967475 & 5.68 \mathrm{e}-013 \\ \text { Optimization terminated successfully: } & & \\ \text { Search direction less than 2*options.Tolx } & \\ \text { | } & \end{array}$

Fig. 15 E-SURGE: The Output during the RUN step shows that only 10 iterations are needed for convergence and that the error due to numerical integration is small (lower than $10^{-} 6$ ). 
Beta (Mathematical parameters)

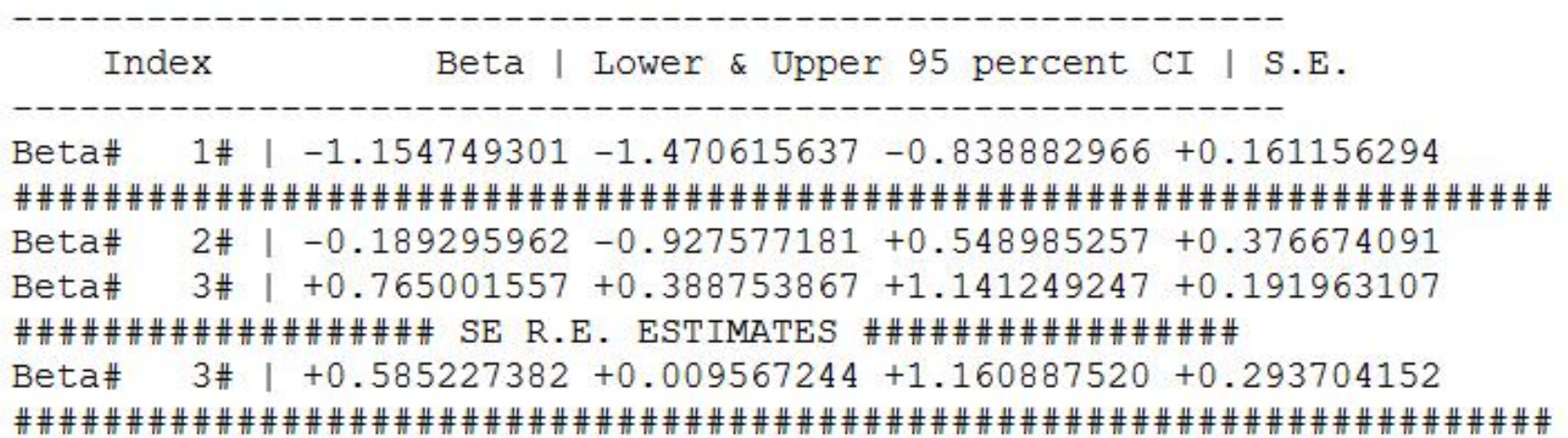

Fig. 16 E-SURGE: Estimates and standard errors for the model $\phi() p.(i+r(g))$. 